\title{
Apogossypolone targets mitochondria and light enhances its anticancer activity by stimulating generation of singlet oxygen and reactive oxygen species
}

\author{
Zhe-Yu Hu ${ }^{1,2,5}$, Jing Wang ${ }^{1,2}$, Gang Cheng ${ }^{3}$, Xiao-Feng Zhu ${ }^{1,2}$, Peng Huang ${ }^{3}$, \\ Dajun Yang ${ }^{4}$, and Yi-Xin Zeng ${ }^{1,2}$
}

\section{Abstract}

Apogossypolone (ApoG2), a novel derivative of gossypol, has been shown to be a potent inhibitor of antiapoptotic Bcl-2 family proteins and to have antitumor activity in multiple types of cancer cells. Recent reports suggest that gossypol stimulates the generation of cellular reactive oxygen species (ROS) in leukemia and colorectal carcinoma cells; however, gossypol-mediated cell death in leukemia cells was reported to be ROS-independent. This study was conducted to clarify the effect of ApoG2-induced ROS on mitochondria and cell viability, and to further evaluate its utility as a treatment for nasopharyngeal carcinoma (NPC). We tested the photocytotoxicity of ApoG2 to the poorly differentiated NPC cell line CNE-2 using the ROS-generating TL/10 illumination system. The rapid ApoG2-induced cell death was partially reversed by the antioxidant $N$-acetyl-L-cysteine (NAC), but the ApoG2-induced reduction of mitochondrial membrane potential (MMP) was not reversed by NAC. In the presence of TL/10 illumination, ApoG2 generated massive amounts of singlet oxygen and was more effective in inhibiting cell growth than in the absence of illumination. We also determined the influence of light on the anti-proliferative activity of ApoG2 using a CNE-2-xenograft mouse model. ApoG2 under TL/10 illumination healed tumor wounds and suppressed tumor growth more effectively than ApoG2 treatment alone. These results indicate that the ApoG2-induced CNE-2 cell death is partly ROS-dependent. ApoG2 may be used with photodynamic therapy (PDT) to treat NPC.

Key words Apogossypolone, mitochondria, singlet oxygen, reactive oxygen species, photodynamic therapy

\section{Introduction}

Nasopharyngeal carcinoma (NPC) is an epithelial squamous cell carcinoma that is endemic in Southeast Asia and parts of the Mediterranean and Northern Africa ${ }^{[1]}$. Cisplatin (DDP) combined with 5-fluorouracil (5-FU) is the first-line chemotherapy for NPC, but toxicity to

\footnotetext{
Authors' Affiliations: 'State Key Laboratory of Oncology in South China, Guangzhou, Guangdong 510060, P. R. China; 'Department of Experimental Research, Sun Yat-sen University Cancer Center, Guangzhou, Guangdong 510060, P. R. China; ${ }^{3}$ Department of Molecular Pathology, The University of Texas MD Anderson Cancer Center, Houston, Texas 77030, USA; ${ }^{4}$ Ascenta Therapeutics Incorporation, Malvern, Pennsylvania 19355, USA; ${ }^{5}$ The First Hospital of Changsha City, Changsha, Hunan 410005, P. R. China.

Corresponding Author: Yi-Xin Zeng, 'State Key Laboratory of Oncology in South China, Guangzhou, Guangdong 510060, P. R. China; ${ }^{2}$ Department of Experimental Research, Sun Yat-sen University Cancer Center, Guangzhou, Guangdong 510060, P. R. China. Tel: +86-2087343333; Fax: 86-20-87343170; Email: zengyx@sysucc.org.cn.
}

normal tissues limited their use at high-doses in NPC patients ${ }^{[2]}$. Previously, we have shown that apogossypolone (ApoG2), a derivative of gossypol, can potently kill NPC cells and work synergistically with DDP to induce cell death $^{[3,4]}$.

Moderate oxidative stress is associated with cancer development ${ }^{[5]}$, but excessive accumulation of cellular reactive oxygen species (ROS) damages DNA, RNA, and proteins, leading to apoptotic or necrotic cell death ${ }^{[6]}$. Multiple chemotherapeutic agents, such as anthracyclines, alkylating agents, epipodophyllotoxins, and camptothecin, can generate high levels of ROS. The phenolic compound gossypol has also been found to elevate ROS in human colorectal carcinoma cells ${ }^{[7]}$ and has potent activity against multiple cancers. Recently, gossypol was shown to stimulate superoxide generation in chronic lymphocytic leukemia (CLL) cells; however, when CLL cells $\mathrm{w}$ ere treated with an antioxidant compound, $\quad \mathrm{N}$-acetyl-L-cysteine (NAC), the gossypol-induced cell death was not blocked, suggesting that gossypol-mediated cell death was ROS-independent ${ }^{[8]}$. 
The killing effect of ROS accumulation in chemotherapy has been mostly neglected by scientists and physicians, and therefore, cancer therapies harnessing the effects of ROS byproducts are poorly developed.

Mitochondria, organelles that generate most of the cell's energy supply, are both a source and a target of ROS. Our previous study has demonstrated that mutations in mitochondrial DNA may be involved in the development and progression of NPC ${ }^{[9]}$. Among the published 1013 mitochondrial proteins [10], the $\mathrm{Bcl}-2$ family, a group of proteins that govern mitochondrial outer membrane permeabilization and control the release of cytochrome $\mathrm{c}$. Without $\mathrm{Bcl}-2$, mitochondria are more vulnerable to proapoptotic signals and toxic substances, such as ROS. In NPC tissue, Bcl-2 is highly expressed and its up-regulation plays a role in NPC tumorigenesis ${ }^{[11]}$. A number of genetic and pharmacologic approaches have been developed to antagonize $\mathrm{Bcl}-2$ in cancer cells ${ }^{[12-14]}$. ApoG2, a small molecular inhibitor of Bcl-2 family proteins, is the oxidation product of gossypol and contains two aromatic hydrocarbon quinone groups. Quinones are of great photobiological importance and serve as electron acceptors in photosynthesis. Quinones undergo reversible enzymatic reduction and oxidation, and form semiquinones or oxygen radicals in cells. Amtitumor quinones, such as adriamycin, mitomycin $\mathrm{C}$ and mitozantrone can form semiquinone radicals in vivo and their antitumor effect is always related to the cytotxicity of oxygen radicals. Thus, if ApoG2 can both inhibit $\mathrm{Bcl}-2$ proteins and produce destructive ROS that would undoubtedly damage mitochondria, it may be a useful therapeutic that can boost cancer cell death.

An important clinical use for ROS in cancer is photodynamic therapy (PDT) ${ }^{[15]}$. During PDT, a photosensitizer is administered to the patient and then the target tissue is exposed to light at a wavelength suitable for exciting the photosensitizer. Under illumination, the photosensitizer is excited to a singlet state and its energy is transferred to a nearby oxygen molecule to create a very aggressive chemical species, singlet oxygen, which very rapidly generates free radicals. These photochemical reactions consequently lead to the generation of cytotoxic ROS, including superoxide and hydrogen peroxide. These destructive ROS can kill cells through apoptosis or necrosis. There is a wide array of photosensitizers for PDT, and some, such as Photofrin and Porphyrin, are available commercially. Classical photosensitizers have low toxicity in the dark and high singlet oxygen quantum yield when excited.

Researchers have tried to use PDT to induce cell death and prevent angiogenesis in NPC ${ }^{[16-18]}$. Compared with normal cells, cancer cells have higher levels of ROS and are more sensitive to oxidative stress ${ }^{[6]}$. In addition, nasopharyngoscopy makes direct and local photodynamic therapy possible.

On the basis of these investigations, we hypothesized that ApoG2 can target the mitochondrial outer membrane and generate excessive ROS to damage mitochondria and induce cell death in CNE-2 cells. To further explore its possible use in PDT, we evaluated the ability of ApoG2 to produce singlet oxygen under illumination.

\section{Materials and Methods}

\section{Cells, drugs, and reagents}

The poorly differentiated human NPC cell line CNE-2 was originally obtained from NPC patients ${ }^{[19]}$ and maintained in our laboratory in Dulbecco's modified Eagle's medium (Gibco/BRL, Gaithersburg, MD) supplemented with $10 \%$ fetal bovine serum (Thermo Scientific HyClone, Logan, UT). Cells were incubated in a humidified $5 \% \mathrm{CO}_{2}$ atmosphere at $37^{\circ} \mathrm{C}$. ApoG2, which was supplied by Professor Dajun Yang (Ascenta Therapeutics Inc., Malvern, Pennsylvania), was dissolved in pure dimethyl sulfoxide (DMSO) at a stock concentration of $20 \mathrm{mmol} / \mathrm{L}$ and stored at $-20^{\circ} \mathrm{C}$. The $5-$ (and - 6 - ) - chloromethyl - 2'7' - dichlorodihydrofluorescein diacetate acetyl ester (CM- $\left.\mathrm{H}_{2} \mathrm{DCF}-\mathrm{DA}\right)$ was obtained from Molecular Probes (Eugene, OR). The 3(4,5-dimethylthiazol-2-yl)-2,5-diphenyltetrazolium bromide (MTT) and NAC were purchased from Sigma-Aldrich (St. Louis, MO), and rhodamine (Rho) 123 was purchased from Invitrogen/Molecular Probes (Carlsbad, CA).

\section{Detection of mitochondrial membrane permabilization}

CNE-2 cells treated either with or without $10 \mu \mathrm{mol} / \mathrm{L}$ ApoG2 were incubated with $1 \mathrm{mmol} / \mathrm{L}$ Rho123 for 30 $\mathrm{min}$. Changes in the mitochondrial outer membrane were detected using flow cytometry. Rho123 accumulates in intact mitochondria and scatters when the mitochondrial membrane collapses.

\section{Determination of cellular ROS level}

Cellular ROS content was measured by incubating untreated control and ApoG2-treated CNE-2 cells with 4 $\mu \mathrm{mol} / \mathrm{L} \quad \mathrm{CM}-\mathrm{H}_{2} \mathrm{DCF}-\mathrm{DA}$ for $30 \mathrm{~min}$. Cells were then harvested and washed with phosphate-buffered saline (PBS) twice and analyzed by flow cytometry using a FACSCalibur flow cytometer equipped with the CellQuest Pro software program (BD Biosciences, Franklin Lakes, NJ).

\section{Detection of ApoG2-induced cell death}

Apoptotic and necrotic cells were detected using 
propidium iodide $(\mathrm{PI})$ staining $^{[20]}$. Untreated control and ApoG2-treated CNE-2 cells were harvested, washed twice, suspended in $500 \mu \mathrm{L}$ of PBS supplemented with $10 \mu \mathrm{L}$ of $\mathrm{PI}(0.23 \mathrm{mmol} / \mathrm{L})$. After $10 \mathrm{~min}$ of incubation with $\mathrm{PI}$ at room temperature, the cells were analyzed using flow cytometry. The cells were classified according to the PI signal as either live cells or late apoptotic or necrotic cells. ApoG2-induced apoptosis was also evaluated by 4,6-diamidine-2-phenylindole (DAPI, 2 $\mu \mathrm{g} / \mathrm{mL}$ ) nuclear staining. The morphologic changes of apoptotic nuclei were examined by fluorescence microscopy using a DF480 microscope (Leica Microsystems, Wetzlar, Germany).

\section{Immunoblot and immunoprecipitation analysis}

The proteins were analyzed by immunoblotting and immunoprecipitation as previously described ${ }^{[21]}$ using primary antibodies specific to the voltage-dependent anion channel (VDAC; Cell Signaling Technology, Danvers, MA), Bcl-2 (Santa Cruz Biotechnology, Santa Cruz, CA), Bax (Santa Cruz Biotechnology), LC3 (Sigma-Aldrich), and actin (Sigma-Aldrich). To test whether ApoG2 interacts directly with $\mathrm{Bcl}-2$ and facilitates the binding of Bax to VDAC in CNE-2 cells, cell lysates were incubated with a Bax antibody, affinity ligand, and protein $A / G$ agarose beads at $4^{\circ} \mathrm{C}$ overnight on a rotator. Proteins bound to the beads were eluted with a denaturing elution buffer, separated by SDS-PAGE, and immunoblotted with either $\mathrm{Bcl}-2$ or VDAC antibody.

\section{Photoactivation of ApoG2 and detection of singlet oxygen}

Cells were pre-incubated in medium containing ApoG2 and were placed $10 \mathrm{~cm}$ from an 8.2-W fluorescent lamp (TL/10) for 1-10 $\mathrm{min}$ to activate ApoG2. Since the intermediate product of singlet oxygen reacting with histidine or imidazole can oxidize $N$, $\mathrm{N}$-dimethyl-p-nitrosoaniline (RNO) and make it fade under light at $440 \mathrm{~nm}$, singlet oxygen was detected chemically by monitoring the bleaching of using a singlet oxygen detection kit (Genmed Scientifics, Inc., Shanghai, China).

The procedure was adapted from the published protocol of Phil Haworth and F. Dan Hess ${ }^{[22]}$. Control and ApoG2-treated CNE-2 cells were exposed to TL/10 for 1 , 3 , or $10 \mathrm{~min}$. Cells were then washed and lysed in lysis buffer, and $25 \mu \mathrm{L}$ of RNO and $25 \mu \mathrm{L}$ of each sample (2 $\mu \mathrm{g} / \mu \mathrm{L}$ ) were added to a $200 \mu \mathrm{L}$ reaction mixture and incubated for $40 \mathrm{~min}$ at $30^{\circ} \mathrm{C}$. Next, the absorption at 440 $\mathrm{nm}$ was measured using a spectrophotometer (Thermo Multiskan MK3; Thermo Fisher Scientific, Waltham, MA). The cellular concentration of singlet oxygen was calculated by the method ${ }^{[23]}$ using the following equation: ${ }^{1} \mathrm{O}_{2}[\mu \mathrm{mol} /(\mathrm{L} \cdot \mathrm{g})]=$ (the absorbance value of sample at $440 \mathrm{~nm} \times 10) /(34.4 \times 0.6 \times$ protein concentration of the sample), where 10 is the diluent's fold of each sample, 34.4 is the absorption coefficient per millimole, and 0.6 is the diameter of the 96-well ELISA palate.

\section{Determination of cell viability}

Cell viability was assessed using an MTT assay. CNE-2 cells were plated in 96-well culture clusters (Costar, Cambridge, MA) at a density of 10 000-15000 cells $/ \mathrm{mL}$. Serial dilutions of ApoG2 were prepared at various concentrations from a stock solution. All experimental concentrations of ApoG2 were prepared in triplicate. Cells were exposed to TL/10 for $10 \mathrm{~min}$ or for fractioned time points. The percent absorbance of the ApoG2-treated cells relative to the DMSO-treated control cells $(<0.1 \%$ DMSO) was plotted as a linear function of the drug concentration. The anti-proliferative effect of ApoG2, with or without illumination, on NPC cells was measured as the percentage of viable cells relative to the DMSO-treated control cells. The $50 \%$ inhibition concentration of ApoG2 was defined as the concentration of the drug required to achieve $50 \%$ growth inhibition relative to control populations.

\section{Assay of in vivo antitumor activity of ApoG2 with TL/10}

As described in our previous study ${ }^{[3]}, \mathrm{CNE}-2$ xenografts formed by subcutaneous injection of four-week-old athymic nude (nu/nu) mice (Animal Center of Guangdong, Guangzhou, China) were removed from the mice and mechanically dissected into equal pieces. The xenografts were then transplanted into the right back area of another group of nude mice. When the tumors became palpable $\left(\sim 0.1 \mathrm{~cm}^{3}\right)$, the mice were randomly divided into three groups (6 mice in each group). To test the phototoxicity of ApoG2, the surface skin tissue of CNE-2 xenografts were shaved using a surgical scissor. A superficial wound $(2 \mathrm{~mm} \times 0.5 \mathrm{~mm})$ was made in the xenograft of each mouse as described prevously in tumor wounding model of colon adenocarcinoma ${ }^{[24]}$. Since the CNE-2 xenograft tum ors grew fast, the CNE-2 cells quickly invaded the superficial tissues and the wound was unable to heal. The two treatment groups received ApoG2 daily $(60 \mathrm{mg} / \mathrm{kg}$, intravenously +30 $\mathrm{mg} / \mathrm{kg}$ topically to cover the tumor wound), and one of the two groups was also treated with TL/10 illumination (10 min daily). For drug administration, PEG 400, cremophor EL, and water (20:3:77) were used to form a solution of ApoG2. The control group received an equal volume of solvent intravenously. The mice were monitored daily for tumor growth, wound size, and their 
body weight. Tumor size $\left(\mathrm{mm}^{3}\right)$ was calculated with the following formula: $4 \pi / 3 \times($ length $/ 2) \times(\text { width } / 2)^{2}$. The tumor growth inhibition ratio $(\mathrm{T} / \mathrm{C} \%)$ was calculated by dividing the average weight of the mouse in the treatment group by that of the mouse in the control group.

\section{Statistical analysis}

All analyses to compare the significance of measured levels were completed using the unpaired $t$-test by SPSS 16.0 software. Significance levels were set at $P<0.05$.

\section{Results}

\section{ApoG2 targeted mitochondria and damaged the mitochondrial outer membrane in CNE-2 cells}

The functional integrity of mitochondrial membranes is typically assessed by flow cytometry after cells are labeled with Rho123, a membrane potential-sensitive dye that accumulates in intact mitochondria and emits red fluorescence. A decrease in this fluorescence indicates a decrease in the mitochondrial membrane potential (MMP), whereas the loss of this fluorescence suggests that the mitochondrial membrane has collapsed, which occurs in the early stages of cell death. As compared with untreated cells, ApoG2 caused a rapid and significant decrease (by about $80 \%$ and $86 \%$ ) in the Rho123 signal in CNE-2 cells after 0.5 and $1 \mathrm{~h}$, respectively (Figure $1 A-C$ ). This shift in the right peak of the Rho123 signal represented a decrease in the MMP of the intact mitochondrial membrane. In addition, ApoG2 also caused the time-dependent loss of the Rho123 signal in $32 \%$ and $41 \%$ of CNE-2 cells after 30 min and $1 \mathrm{~h}$, respectively.

Although ApoG2 has been identified as a novel inhibitor of the antiapoptotic Bcl-2 family proteins, its intracellular effect on the mitochondrial outer membrane and transmembrane pore has remained unclear. Because VDAC is one of the most abundant proteins on the outer mitochondrial membrane ${ }^{[25]}$ and directly interacts with Bax to form a large pore that is permeable to cytochromec ${ }^{[26,27]}$, we tested whether VDAC was involved in the ApoG2-induced damage of the mitochondrial membrane in CNE-2 cells. We examined the interaction of Bax with either $\mathrm{Bcl}-2$ or VDAC by immunoprecipitation analysis using a Bax-specific antibody. VDAC was bound to Bax following ApoG2-mediated inhibition of $\mathrm{Bcl}-2$, whereas the total expression levels of VDAC, Bax, and Bcl-2 were not affected by ApoG2 treatment (Figure 1). These results suggest that ApoG2 facilitates the interaction of Bax with VDAC and increases the permeability of the mitochondrial outer membrane by targeting the mitochondria and binding to $\mathrm{Bcl}-2$.

\section{ApoG2 induced ROS accumulation in CNE-2 cells}

ApoG2 not only increased the permeability of the mitochondrial outer membrane but also induced the collapse of the mitochondria in $0.5-1 \mathrm{~h}$ (Figure 1), which happened in the early stage of cell death. To determine the effect of ApoG2 on ROS accumulation in CNE-2 cells, we used the fluorescent probe $\mathrm{CM}-\mathrm{H}_{2} \mathrm{DCF}-\mathrm{DA}$, which specifically detects cellular hydrogen peroxide content, to measure the cellular ROS level in CNE-2 cells. ApoG2 treatment caused significant increases (1.4-, 2.9-, and 14.0-fold) in ROS levels at 0.5, 1.0, and $3.0 \mathrm{~h}$, respectively, as measured by flow cytometry (Figure 2A).

To investigate the cause-and-effect relationship between excessive ROS levels and mitochondrial damage, we examined the effect of NAC, an antioxidant compound that eliminates hydrogen peroxide by increasing cellular glutathione (GSH) levels, on ApoG2-induced mitochondrial damage. NAC significantly reduced ROS levels in ApoG2-treated cells (Figure 2B) and partly reversed the ApoG2-induced loss of the Rho-123 fluorescence signal (from $41.63 \%$ to $28.27 \%$ ) in cells with collapsed mitochondria (Figures 1C and 2D); however, NAC did not reverse the ApoG2-induced decrease in the MMP in cells with intact mitochondria (Figures 2C and 2D). This suggests that the ApoG2-induced mitochondrial collapse may be partially caused by the accumulation of ROS in CNE-2 cells. However, the decrease in the MMP in cells with intact mitochondria did not depend on the cellular ROS level.

\section{ROS generation facilitated ApoG2-induced cell death}

The ability of ApoG2 to cause severe ROS-mediated damage in mitochondria prompted us to test its effectiveness in killing CNE-2 cells. Previously, we had shown that ApoG2 induced apoptotic cell death in CNE-2 cells. But in some cancer cells that are absent of apoptosis because of mutations in critical molecules of the apoptotic machinery, autophagic cell death is an alternative form of cell death ${ }^{[28]}$. During autophagy, a ubiquitin-like microtubule-associated protein 1 light chain 3-I (LC3-I) is converted to a phosphatidylethanolamine (PE)-conjugated LC3-II form, and the amount of LC3-II correlates with the extent of autophagosome formation ${ }^{[29]}$. As shown in Figure 3A, $10 \mu \mathrm{mol} / \mathrm{L}$ ApoG2 caused a significant conversion of LC3-I to LC3-II after $24 \mathrm{~h}$. Excessive ROS accumulation also caused apoptotic cell death in CNE-2 cells, which was partly reversed by the antioxidant NAC (Figures 3B and 3 C). 


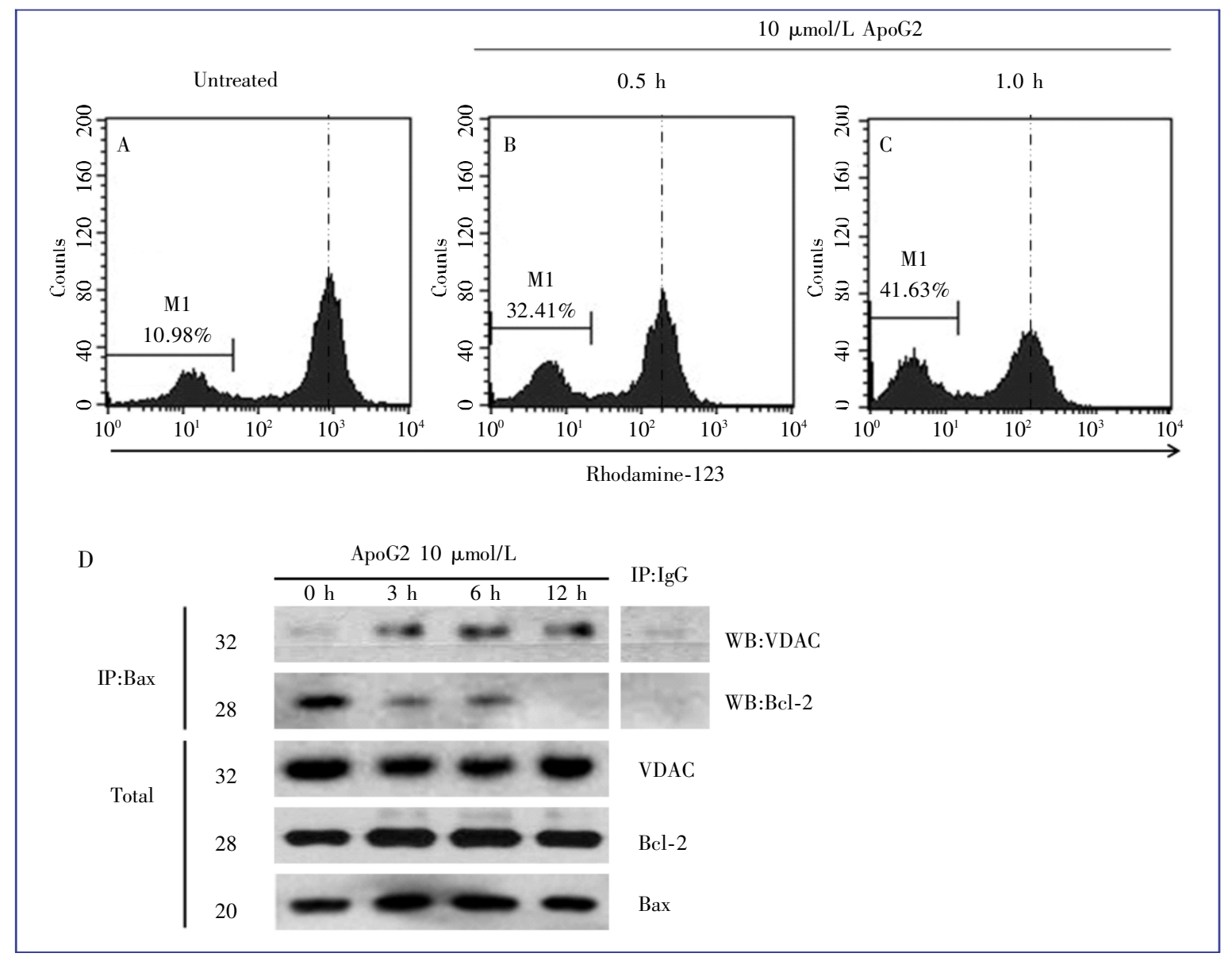

Figure 1. Ten micromolar ApoG2 modulates the mitochondrial membrane potential in CNE-2 cells. Untreated CNE-2 cells (A) and CNE-2 cells treated with ApoG2 for either 30 min $\quad$ (B) or $1 \mathrm{~h}$ (C) were stained with Rho-123, and the red Rho-123 fluorescent signals were detected by flow cytometry. In the diagrams, the right peak of the Rho-123 signal indicates the MMP signal in cells with an intact mitochondrial membrane, whereas the appearance of the left signal peak (M1) indicates the subpopulation of cells that have lost their mitochondrial transmembrane potential and have collapsed mitochondria. Each diagram is representative of three independent experiments. D, Western blot analysis of ApoG2-treated CNE-2 cells immunoprecipitated with an anti-Bax antibody shown in the top panel. Bottom panel shows Western blotting with anti-VDAC, anti-Bcl-2, and anti-Bax antibodies to detect their total expression levels in ApoG2-treated cells.

We also detected cell death using propidium iodide (PI). About $10 \%$ of untreated cells, were PI-positive, whereas more than $37 \%$ of the CNE-2 cells treated with $10 \mu \mathrm{mol} / \mathrm{L}$ ApoG2 were PI-positive after $12 \mathrm{~h}$ (Figure 3D). NAC significantly reduced the percentage of PI-positive cells (to $17 \%$ ) in ApoG2-treated CNE-2 cells (Figure 3D), indicating that elimination of ROS by NAC could reverse the ApoG2-induced cell death. In summary, these findings strongly suggest that the ApoG2-induced cell death is at least partially mediated by the oxidative stress pathway.

\section{Light stimulated the generation of singlet oxygen in ApoG2-treated CNE-2 cells}

ApoG2 is the oxidation product of gossypol and has two aromatic hydrocarbon quinone groups. Because quinones serve as electron acceptors in photosynthesis, we hypothesized that the quinone structure of ApoG2 was light sensitive and could generate singlet oxygen and ROS in a photochemical reaction (Figure 4A). To choose a safe and efficient illumination system for investigating the sensitivity of ApoG2 to light, we examined the absorption spectrum of ApoG2 using an ultraviolet spectrophotometer (Shimadzu UV-2450, Japan). Three absorption peaks were detected for ApoG2 at 215.5-, 282.5- and 380-nm wavelengths. To eliminate direct damage of the cells by light, we chose the fluorescent lamp, TL/10 (Philips), which emitted long-wave UVA radiation between 350 and $400 \mathrm{~nm}$, with a maximum at $372 \mathrm{~nm}$ near the visible spectrum (Figure $4 \mathrm{~B}$ ). Its radiation power was $8.2 \mathrm{~W}$ and it was unable to stimulate a notable increase in singlet oxygen after 10 min when placed $10 \mathrm{~cm}$ from the cells (Figure 4C).

The photosensitization process occurred as oxidation through formation of singlet oxygen. Analysis of the 


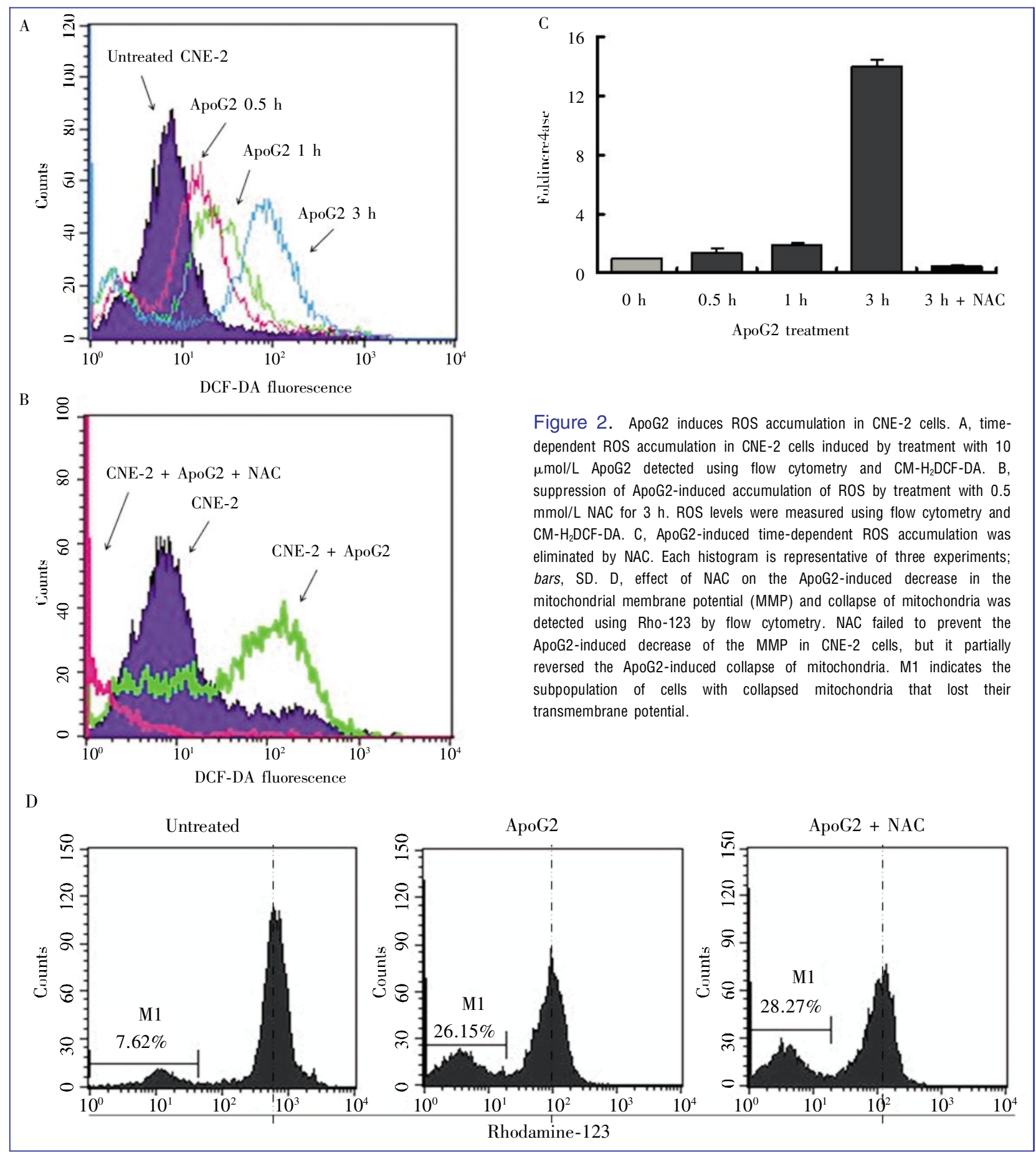

cellular level of singlet oxygen showed a time-dependent generation of singlet oxygen induced by $\mathrm{TL} / 10$ radiation and ApoG2 treatment (Figure 4C). Although ApoG2 could trigger a moderate increase in singlet oxygen without $\mathrm{TL} / 10$ radiation, the photoactivation process was required to achieve the greatest oxidative toxicity in cancer cells. As shown in Figure 4D, CNE-2 cells were pre-incubated in medium containing ApoG2 in the dark for $10 \mathrm{~min}$, followed by exposure to TL/10 for either 1 or $10 \mathrm{~min}$ to activate ApoG2 and subsequent re-incubation in the dark for another $5 \mathrm{~min}$. The control cells were incubated with an equivalent concentration of ApoG2 in the dark for either 16 or $25 \mathrm{~min}$. Compared with treatment of ApoG2 alone, the extra radiation from the $\mathrm{TL} / 10$ lamp for either 1 or $10 \mathrm{~min}$ stimulated a significantly higher cellular concentration of singlet 


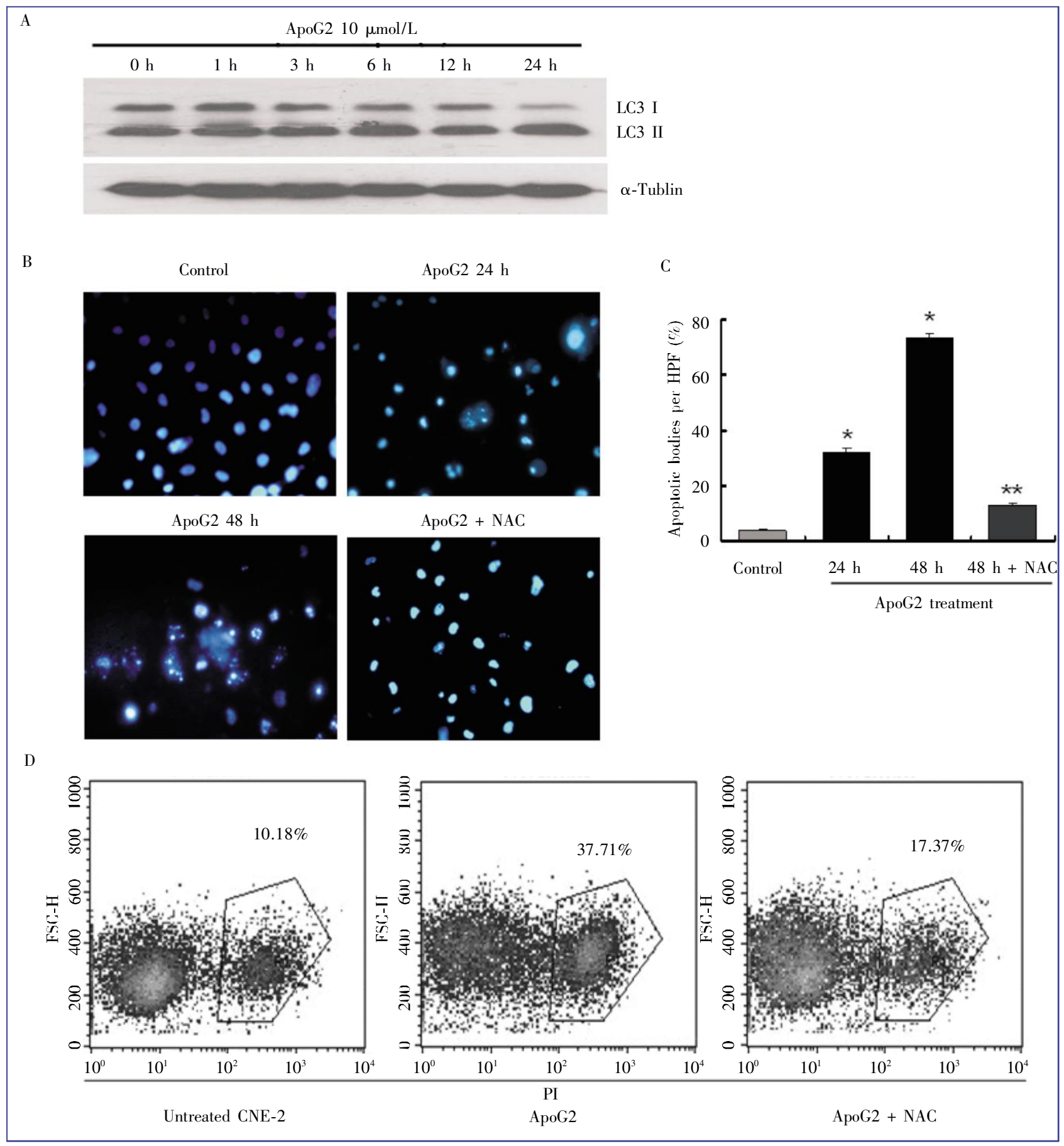

Figure 3. ApoG2-induced cell death is facilitated by excessive accumulation of ROS. A, CNE-2 cells were treated with $10 \mu$ mol/L ApoG2 for $1,3,6,12$, and 24 h. Cell lysates were examined by Western blot analysis using anti-LC3 (top) and anti-tubulin (bottom) antibodies. B, staining with DAPI shows characteristic features of apoptosis in ApoG2-exposed CNE-2 cells. CNE-2 cells were treated either with $10 \mu \mathrm{mol} / \mathrm{L}$ ApoG2 for 24 and $48 \mathrm{~h}$ or with ApoG2 and $0.5 \mathrm{mmol} / \mathrm{L} \mathrm{NAC}$ for $48 \mathrm{~h}$; thereafter, cells were stained with DAPI and detected by fluorescence microscopy. Cells treated with DMSO $(0.1 \%)$ served as the control. C, the number of apoptosis-characteristic DAPI-stained nuclei per $\times 40$ high-power field (HPF) is indicated. Numbers indicate the average for five HPFs per sample; each histogram is representative of three experiments; bars, SD. ${ }^{*}, P<0.05$, statistically significant increase in the number of apoptotic bodies compared with DMSO-treated control cells; ${ }^{* *}, P<0.05$, statistically significant decrease in the number of apoptotic bodies compared with cells treated with ApoG2. D, reversal of ApoG2-induced cell killing by NAC. Necrotic and late apoptotic cell deaths in CNE-2 cells were detected using PI staining and flow cytometry. The cells were classified according to the PI signal as either live cells (in histograms, left population with low PI signals) or late apoptotic or necrotic cells (the gated right population with high PI signals). The gated population indicates the dead cells with high PI signals. 
A Aromatic hydrocarbon

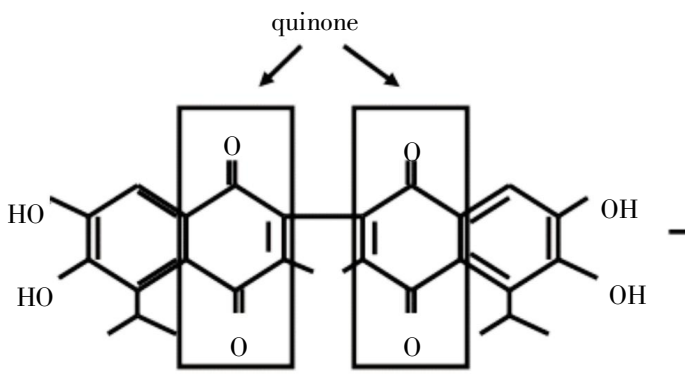

Photochemical reaction

Reactive oxygen

species

ApoG2

B

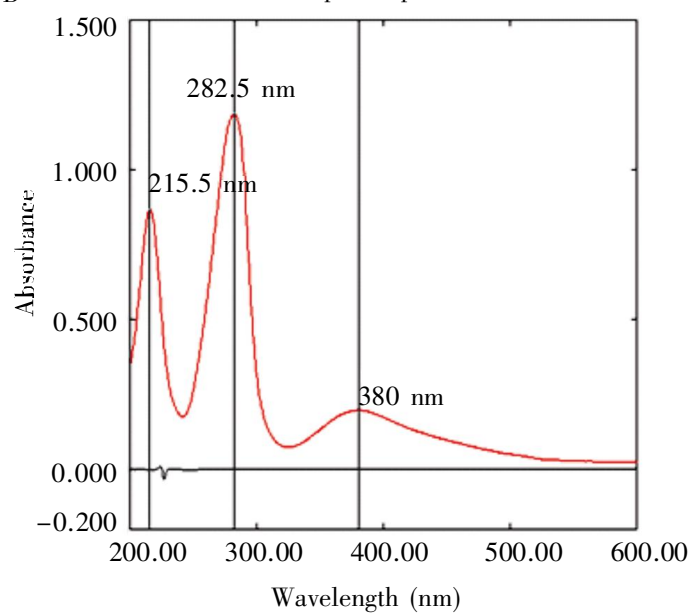

C

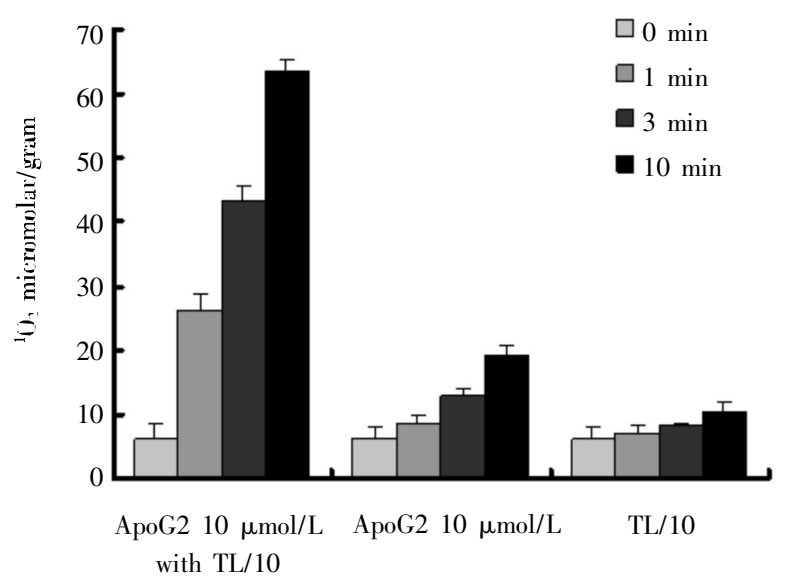

$\mathrm{TL} / 10$

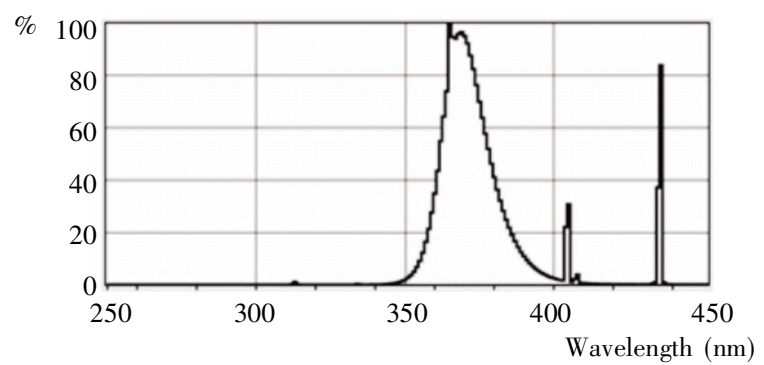

Spectral power distribution

D

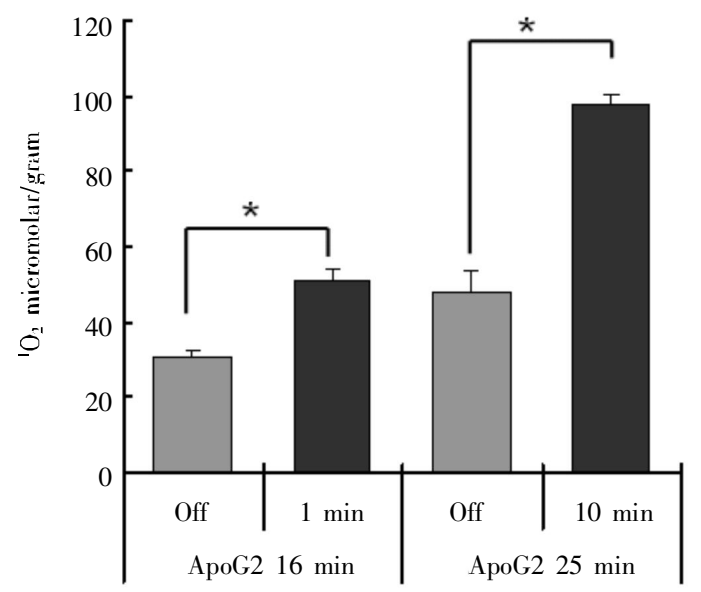

Figure 4. Light induces the generation of singlet oxygen by ApoG2 in CNE-2 cells. A, the chemical structure of ApoG2. B, ultraviolet (UVA) absorption spectrum of ApoG2. Three absorption peaks at 215.5, 282.5, and $380 \mathrm{~nm}$ are indicated in the left diagram. Right, TL/10 fluorescent lamp emits long-wave UVA radiation between 350 and $400 \mathrm{~nm}$ with a maximum at $372 \mathrm{~nm}$. C, CNE-2 cells were treated as indicated for $0,1,3$, or $10 \mathrm{~min}$. The time-dependent generation of singlet oxygen induced by TL/10 radiation in the presence of ApoG2 was detected using the luminescent substrate $N$, $N$-Dimethyl-p-nitrosoaniline (RNO). D, CNE-2 cells were pre-incubated in medium containing ApoG2 in the dark at $37^{\circ} \mathrm{C}$ for $10 \mathrm{~min}$, followed by exposure to the $\mathrm{TL} / 10$ lamp, which was placed $10 \mathrm{~cm}$ from the sample, for either 1 or 10 min to activate ApoG2. Control samples were incubated with an equivalent concentration of ApoG2 without exposure to the TL/10 lamp. Each histogram is representative of three experiments; bars, SD. ${ }^{*}, P<0.001$, statistically significant increase in the ${ }^{1} \mathrm{O}_{2}$ level compared with control cells. 
oxygen $(P<0.001)$.

\section{Light enhanced the anti-proliferative activity of ApoG2 in CNE-2 cells}

Because TL/10 radiation increased the level of singlet oxygen, we expected to see a corresponding increase in the anti-proliferative activity of ApoG2 after exposure to TL/10 radiation. The cytotoxicity and photocytotoxicity of ApoG2 were examined using the MTT assay. CNE-2 cells were exposed to $0.16-40$ $\mu \mathrm{mol} / \mathrm{L} \mathrm{ApoG} 2$ for 24,48 , and $72 \mathrm{~h}$. As shown in Figure $5 \mathrm{~A}$, exposure to ApoG2 alone resulted in a time- and dose-dependent inhibition of cell viability. After $10 \mathrm{~min}$ of TL/10 illumination, ApoG2 inhibited cell growth more effectively than ApoG2 alone, with $\mathrm{IC}_{50}$ values of $(4.17 \pm$ $0.87)$ and $(1.31 \pm 0.28) \mu \mathrm{mol} / \mathrm{L}$, respectively, after $72 \mathrm{~h}$ (Figure 5B).

Because the half-life of reactive oxygen is too short ( 25-30 $\mathrm{min}$ in air and $35 \mathrm{~min}$ in water) and its toxicity will not persist for a long time in the cell, we hypothesized that fractionation of the photoradiation might result in a greater injury accumulation during illumination. In MTT assay, CNE-2 cells were incubated with $0.16-40 \mu \mathrm{mol} / \mathrm{L}$ ApoG2 and, $30 \mathrm{~min}$ later, cells were exposed to either TL/10 radiation for 10 min once a day or to TL/10 radiation that was fractioned across either 3 or 6 illumination procedures. Figure $5 \mathrm{C}$ showed the effects of ApoG2 with single and split doses of photoradiation. Compared with the single 10-min radiation dose, the 3-min, 20-sec split dose given 3 times did not significantly inhibit cell growth $(P=0.23)$; however, the 1-min, 40-sec split dose given 6 times was more effective than the single $10 \mathrm{~min}$ dose in inhibiting cell growth $(P<0.05)$. The $\mathrm{IC}_{50}$ values were $(1.31 \pm$ $0.28), \quad(0.90 \pm 0.07)$, and $(0.47 \pm 0.04) \mu \mathrm{mol} / \mathrm{L}$, respectively.

\section{Light enhanced the ApoG2-mediated reduction in size of superficial wound patch and suppressed CNE-2 xenograft growth in nude mice}

Since light boosted the ROS-mediated cell death in CNE-2 cells in vitro, we wanted to test its effectiveness in suppressing the growth of CNE-2 tumor xenografts in vivo. Because light at $385 \mathrm{~nm}$ does not penetrate deeply into the center of tumors, we used a tumor superficial wounding model to test the phototoxicity of ApoG2 in vivo. As shown in Figure 6A, TL/10 illumination potently enhanced the ability of ApoG2 to heal the tumor wound. Compared with the control group, ApoG2 greatly reduced the size of the wound patch, and when TL/10 illumination was added, the wound patches on the tumor surface were almost eradicated (Figure 6B). To measure the effects of ApoG2 on tumor growth, we measured the tumor growth inhibition ratio, $\mathrm{T} / \mathrm{C} \%$. The $\mathrm{T} / \mathrm{C} \%$ ratio was $72 \%$ for the group treated with ApoG2 alone and $44 \%$ for the group treated with both ApoG2 and TL/10 illumination (Figure $6 \mathrm{C}$ ). Figure $6 \mathrm{D}$ shows the tumor volumes $\left(\mathrm{cm}^{3}\right)$ of mice treated with ApoG2 alone and ApoG2 plus TL/10 illumination. No severe toxicity was observed, although some mice exhibited transient agitation at the time of drug injection. Furthermore, we did not detect a significant reduction in the body weight of the mice.

\section{Discussion}

Regulation of outer mitochondrial membrane permeability is a complex process involving both the regulation of intact organelles by $\mathrm{Bcl}-2$ family proteins and the regulation of the collapsed mitochondrion ${ }^{[30,31] .}$ VDAC is one of the most abundant proteins in the outer mitochondrial membrane ${ }^{[25]}$ and directly interacts with Bax to form a large pore that is permeable to cytochrome $\mathrm{C}^{[26,2]}$. Bcl-2 and Bcl-XL can incorporate into the mitochondrial outer membrane, resulting in closure of VDAC and inactivation of the adenine nucleotide translocator ${ }^{[32]}$. As an inhibitor of $\mathrm{Bcl}-2$ family antiapoptotic proteins, ApoG2 is previously reported to directly block the binding of $\mathrm{Bcl}-2$ and $\mathrm{Bax}^{[3,33]}$. Here, we showed that by blocking the interaction between $\mathrm{Bax}$ and $\mathrm{Bcl}-2$, ApoG2 facilitated the interaction of Bax with VDAC, leading to a significant decrease in the MMP. In addition, ApoG2 induced the complete loss of the MMP in some cells, indicating that ApoG2 has a toxic effect on mitochondrial outer membrane.

To become cancer cells, cells commonly experience increased ROS generation in response to hormones, viral infection, or oncogenic transformation. ROS play an important role in maintaining the cancer phenotype by altering intracellular signaling, stimulating cell growth, and causing genetic instability ${ }^{[3436}$. However, in addition to stimulating multiple cell death pathways, high levels of ROS can also sensitize cancer cells to anticancer agents ${ }^{[37,38]}$. Multiple traditional anticancer agents, including anthracyclines and alkylating agents, are found to stimulate massive ROS generation in cancer cells ${ }^{[39]}$. Several groups have reported that gossypol can affect the cellular redox status ${ }^{[7,8]}$, and gossypol-induced cell death occurs through a ROS-dependent mitochondrial pathway in human colorectal carcinoma cells ${ }^{[7]}$. ApoG2 is the oxidation product of gossypol and contains two para-quinone moieties, making this electrophilic compound very reactive to cellular nucleophiles and enabling it to induce oxidative stress in cells. The ROS level is lower in CNE-2 cells than the nasopharyngeal epithelial cell line NP69, making the former more tolerant to the ROS-inducing compound $\beta$-phenylethyl 

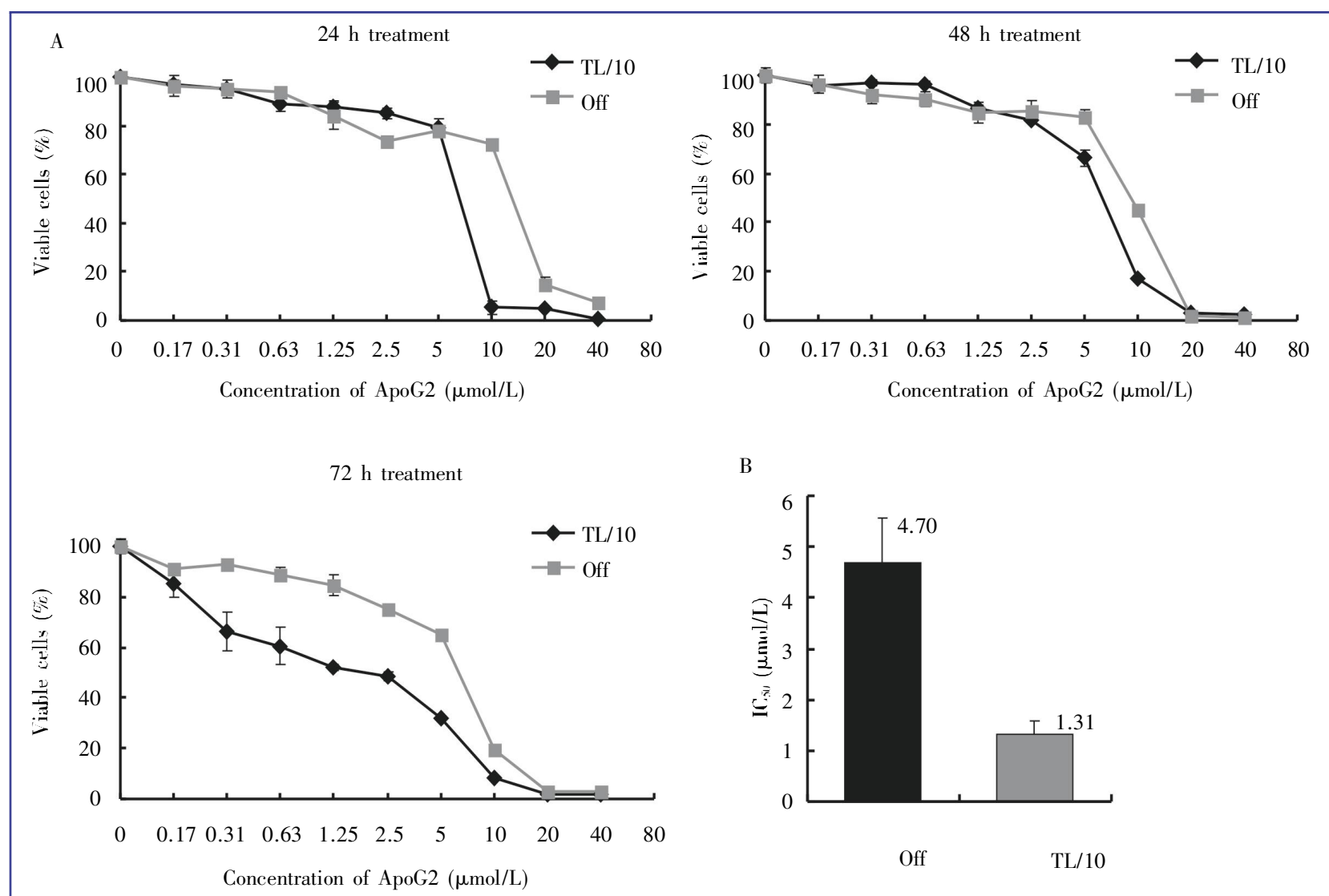

B

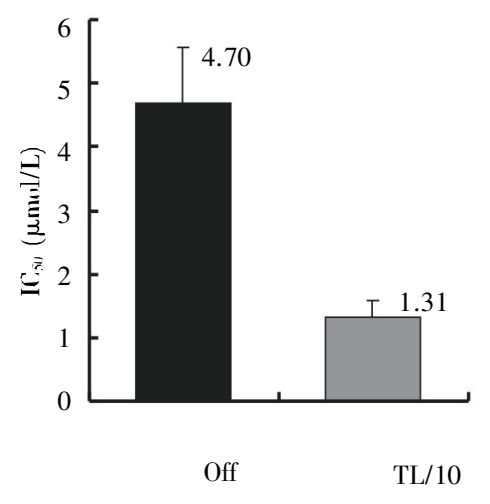

C

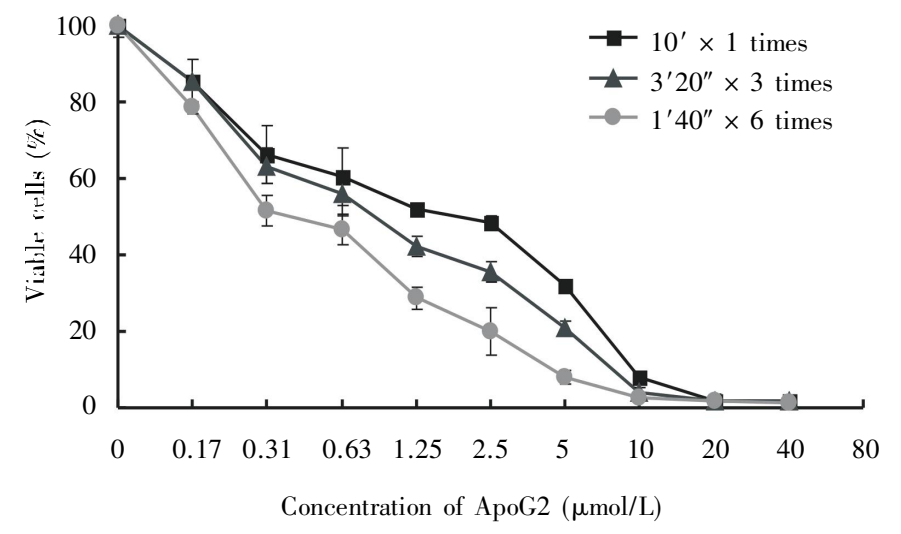

$\mathrm{D}$

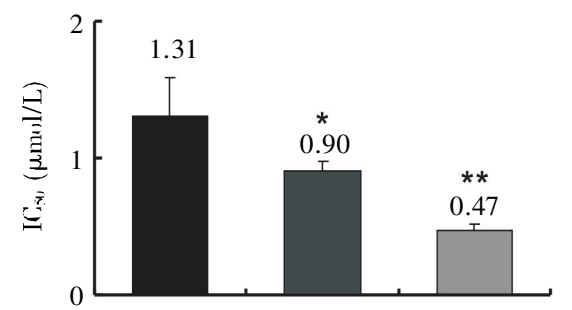

$10^{\prime} \times 1$ times $3^{\prime} 20^{\prime \prime} \times 3$ times $1^{\prime} 40^{\prime \prime} \times 6$ times $\mathrm{TL} / 10$

Figure 5. Light enhances the anti-proliferative activity of ApoG2 in CNE-2 cells. A, effect of TL/10 illumination on the anti-proliferative activity of ApoG2 in CNE-2 cells. Cells were treated with $0-40 \mu \mathrm{mol} / \mathrm{L}$ ApoG2, with or without TL/10 illumination. Points, average of 3 experiments; bars, SD. B, comparison of the IC 50 values for ApoG2 alone and ApoG2 plus 10 min TL/10 illumination (mean $\pm \mathrm{SD}$ of three experiments; ${ }^{*} P<0.05$ ). C, ApoG2-treated CNE-2 cells were exposed to TL/10 illumination for $10 \mathrm{~min}$ once, $3 \mathrm{~min}$ and $20 \mathrm{~s}$ three times, or $1 \mathrm{~min}$ and $40 \mathrm{~s}$ six times. The effect of the fractioned illumination on the anti-proliferative activity of ApoG2 was measured by the MTT assay (mean $\pm S D$ of three experiments). $D$, comparison of the $I_{50}$ values for the fractioned illumination (mean \pm SD of three experiments; ${ }^{*} P<0.05$ ).

isothiocyanate (data not shown). Nevertheless, the accumulation of ROS might still play a role in ApoG2-induced cell death in CNE-2 cells in the absence of illumination. Indeed, in this study, ApoG2 was shown to damage mitochondria and induce cell death by generating an excessive amount of ROS. Ten micromolar ApoG2 caused a tremendous increase in cellular ROS levels in CNE-2 cells within $3 \mathrm{~h}$ (Figure 2A). The antioxidant compound NAC not only partially reversed the ApoG2-induced cell death (Figure 3D) but 


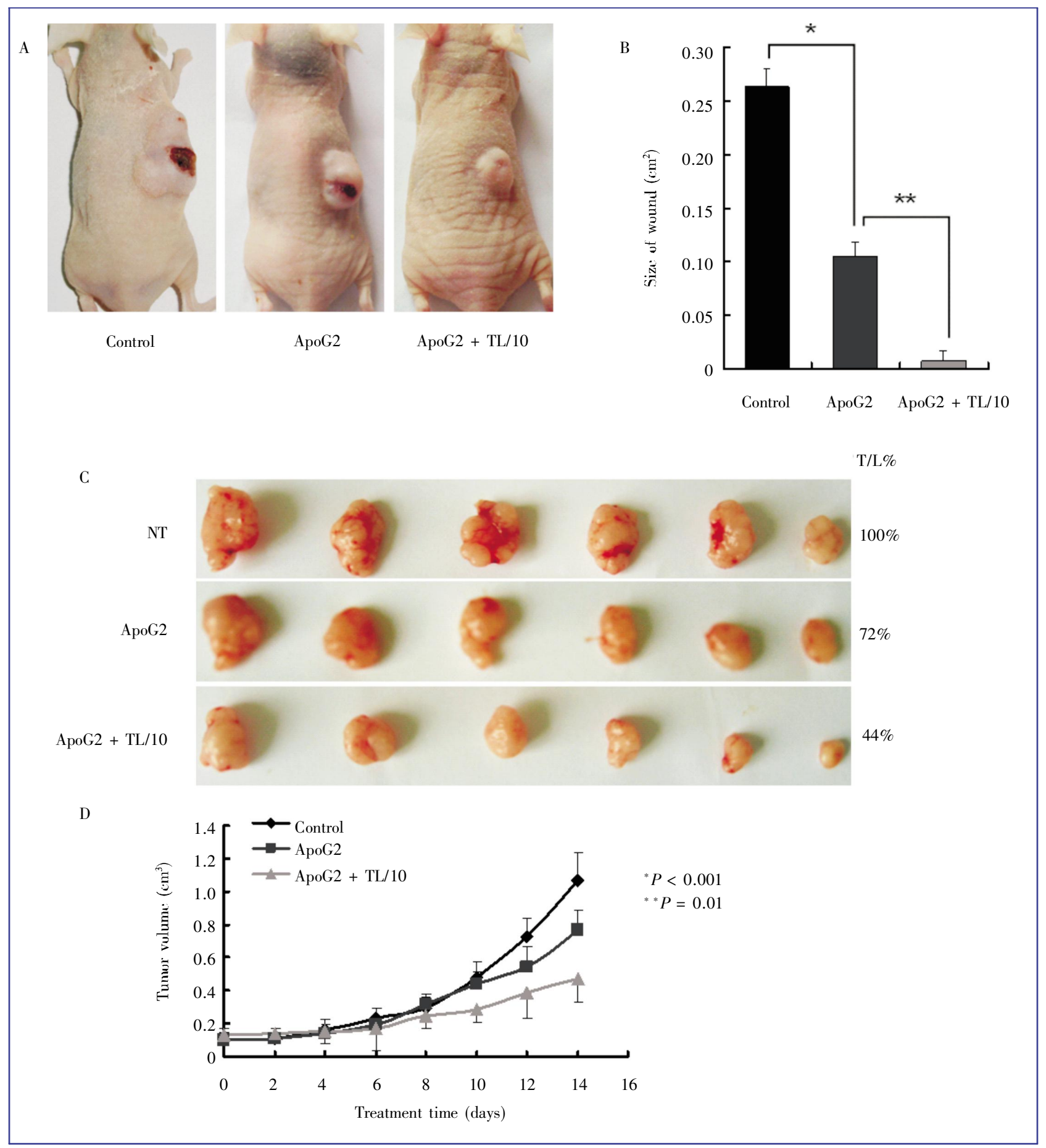

Figure 6. Light enhances the ApoG2-induced inhibition of CNE-2-xenograft tumor growth. A, photographs of nude mice bearing CNE-2 xenografts with a superficial wound. Untreated, ApoG2-treated $(60 \mathrm{mg} / \mathrm{kg}$ intravenously $+30 \mathrm{mg} / \mathrm{kg}$ topically covering the tumor wound, daily), and ApoG2 plus TL/10 illuminationtreated mice are shown. B, compared to the control group, ApoG2 greatly reduced the size of the wounds $\left({ }^{*} P<0.05\right)$. The addition of TL/10 illumination helped ApoG2 to further heal the tumor wounds $\left({ }^{\star *} P<0.05\right)$. Bar heights, average size of tumor wounds; bars, SD. C, photographs of CNE-2 xenografts from untreated, ApoG2-treated, and ApoG2 plus TL/10 illumination-treated groups. The tumor growth inhibition ratio (T/C\%) was calculated by dividing the average weight of the mouse in the treatment group by that of the mouse in the control group. D, inhibition of tumor growth by ApoG2 or ApoG2 plus TL/10 illumination in CNE-2 tumor-bearing nude mice (the values and the bars, means $\pm \mathrm{SD}$ ). Compared to the control group, both ApoG2 alone and ApoG2 plus TL/10 illumination significantly inhibited growth rate of CNE-2 xenografts ( ${ }^{*} P<0.001$ ). Compared to ApoG2 alone, ApoG2 plus TL/10 showed even higher inhibitory effect ${ }^{* *} P=0.01$ ). 
also partially blocked the ApoG2-induced loss of MMP in CNE-2 cells. However, NAC could not reverse the decrease in the MMP in ApoG2-treated CNE-2 cells. These results suggest that ApoG2-induced mitochondrial damage and cell death was caused by both inhibition of antiapoptotic Bcl-2 family proteins and the destructive effect of excessive ROS accumulation.

Beside anticancer chemotherapy compounds, several photo-sensitizers have been developed to kill cancer cells by generating ROS and singlet oxygen in the presence of illumination. Under suitable types of light, the photo-sensitizer is excited into an active state and transfers its energy to nearby oxygen molecules to create singlet oxygen. Singlet oxygen is very active; it can rapidly generate free radicals and destroy cells. ApoG2 has two aromatic hydrocarbon quinone groups. Quinones are of great photobiological importance and play a role in photosynthesis. In addition, the photoreduction of 1,4-benzoquinone and its derivatives, including 1,4-naphthoquinone (NQ) and 2-methyl-1,4-naphthoquinone (MeNQ), is frequently used to generate radicals ${ }^{[40-42]}$. As we have shown in this study, ApoG2 treatment results in a significant increase in the cellular singlet oxygen concentration in CNE-2 cells when illuminated by a TL/10 lamp (Figure 4). These findings prompted us to evaluate the use of ApoG2 as a photo-sensitizer in PDT for the treatment of NPC. In the clinical context, PDT has been successfully used to treat superficial lesions of multiple cancers, such as skin cancer, breast cancer, and head and neck cancers. Although NPC is a solid tumor always located deeply in the pharyngeal recess, a fiberoptic nasopharyngoscope makes direct visualization of tumor tissue and PDT possible. The photo-sensitizer 5-aminolevulinic acid and its hexyl ester have shown promising in vitro PDT results against NPC ${ }^{[43]}$. Hypericin-based PDT also induces NPC tumor necrosis in vivo ${ }^{[44]}$. Recently, temoporfin has also shown a significant benefit to patients with advanced head and neck carcinoma in clinical trials ${ }^{[45]}$. Because body tissue is more transparent at higher wavelengths, photo-sensitizers, including 5-aminolevulinic acid, hypericin, and foscan, have absorption peaks at long wavelengths. ApoG2 has three absorption peaks at wavelengths of 215, 282, and $385 \mathrm{~nm}$ (Figure 4). Even the light at $385 \mathrm{~nm}$, which is near the visible light portion of the spectrum, does not penetrate deeply. Because primary NPC tumors always invade the superficial mucosa of the nasopharynx, and a nasopharyngoscope can directly reach the diseased region, we hypothesized that a nasopharyngoscope with a light beam with a wavelength of $385 \mathrm{~nm}$ might be able to excite the activity of ApoG2 on the surface of the malignant mucosa. Consistent with this hypothesis, ApoG2 treatment with illumination showed higher anti-proliferative activity against CNE-2 cells in vitro (Figure 5 ) and also exhibited higher antitumor activity against NPC xenografts in vivo (Figure 6). Treatment of ApoG2 on the tumor wound with illumination also helped heal the wound. A classical photo-sensitizer should have low dark toxicity and not be harmful to normal tissue in the dark. Though ApoG2 showed high toxicity to NPC in the dark, it is not harmful to normal tissues because normal tissues lack its targets, the antiapoptotic Bcl-2 family proteins. Furthermore, in our in vivo study, ApoG2 treatment did not cause a reduction in body weight or any other side effects in mice. Based on these findings, we believe that PDT may be useful for elevating the antitumor activity of ApoG2 in the treatment of NPC.

Researchers have recently reported that fractioned illumination can improve the outcome in the treatment of precancerous lesions with PDT ${ }^{[46]}$. In NPC radiotherapy, a fractionated radiation dose provides better tumor control ${ }^{[4]}$. Here, we also evaluated the effect of ApoG2 with split-dose photoirradiation using a cytotoxicity assay. As shown in Figure 5, ApoG2 treatment with split-dose photoirradiation resulted in more cell death than single-dose photoirradiation. In conclusion, our study suggests that the eletrophilicity of ApoG2 associated with its quinone moieties provides a basis for developing strategies, such as PDT, to enhance its antitumor activity in NPC cells.

Received: 2010-06-12; revised: 2010-08-09; accepted: 2010-08-12.

\section{References}

[1] McDermott AL, Dutt SN, Watkinson JC. The aetiology of nasopharyngeal carcinoma [J]. Clin Otolaryngol Allied Sci, 2001,26(2):82-92

[2] Henderson S, Rowe M, Gregory C, et al. Induction of bcl-2 expression by Epstein-Barr virus latent membrane protein 1 protects infected B cells from programmed cell death [J]. Cell, 1991, 65(7):1107-1115.

[3] Hu ZY, Zhu XF, Zhong ZD, et al. ApoG2, a novel inhibitor of antiapoptotic $\mathrm{Bcl}-2$ family proteins, induces apoptosis and suppresses tumor growth in nasopharyngeal carcinoma xenografts [J]. Int J Cancer, 2008, 123(10):2418-2429.

[4] Hu ZY, Sun J, Zhu XF, et al. ApoG2 induces cell cycle arrest of nasopharyngeal carcinoma cells by suppressing the c-Myc signaling pathway [J]. J Transl Med, 2009, 7:74.

[5] Behrend L., Henderson G, and Zwacka RM. Reactive oxygen species in oncogenic transformation [J]. Biochem Soc Trans, 2003, 31(Pt 6):1441-1444. 
[6] Pelicano H, Carney D, and Huang P. ROS stress in cancer cells and therapeutic implications [J]. Drug Resist Updat, 2004, $7(2)$ : 97-110.

[7] Ko CH, Shen SC, Yang LY, et al. Gossypol reduction of tumor growth through ROS-dependent mitochondria pathway in human colorectal carcinoma cells [J]. Int J Cancer, 2007,121(8):1670-1679.

[8] Balakrishnan K, Wierda WG, Keating MJ, et al. Gossypol, a BH3 mimetic, induces apoptosis in chronic lymphocytic leukemia cells [J]. Blood, 2008, 112(5):1971-1980.

[9] Shao JY, Li YH, Gao HY, et al. High frequency of common deletion (4981 bp) in mitochondrial DNA in nasopharyngeal carcinoma and its correlation with patient age and clinical stages [J]. Cancer Biol Ther, 2004,3(12): 1275-1276.

[10] Meisinger C, Sickmann A, and Pfanner N. The mitochondrial proteome: from inventory to function [J]. Cell, 2008, 134(1):22-24.

[11] Chou J, Lin YC, Kim J, et al. Nasopharyngeal carcinoma-review of the molecular mechanisms of tumorigenesis [J]. Head Neck, 2008, 30(7):946-963.

[12] Meng Y, Tang W, Dai Y, et al. Natural BH3 mimetic (-)-gossypol chemosensitizes human prostate cancer via Bcl-xL inhibition accompanied by increase of Puma and Noxa [J]. Mol Cancer Ther, 2008, 7(7):2192-2202.

[13] Stein CA, Benimetskaya L, Mani S. Antisense strategies for oncogene inactivation [J]. Semin Oncol, 2005, 32(6):5635-5672.

[14] van Delft MF, Wei AH, Mason KD, et al. The BH3 mimetic ABT-737 targets selective $\mathrm{Bcl}-2$ proteins and efficiently induces apoptosis via Bak/Bax if Mcl-1 is neutralized [J]. Cancer Cell, 2006,10(5): 389-399

[15] Price M, Reiners JJ, Santiago AM, et al. Monitoring singlet oxygen and hydroxyl radical formation with fluorescent probes during photodynamic therapy. Photochem Photobiol, 2009,85:1177-1181.

[16] Xie Y, Wei ZB, Zhang Z, et al. Effect of 5-ALA-PDT on VEGF and PCNA expression in human NPC-bearing nude mice [J]. Oncol Rep, 2009, 22(6):1365-1371.

[17] Yow CM, Mak NK, Leung AW, et al. Induction of early apoptosis in human nasopharyngeal carcinoma cells by mTHPC-mediated photocytotoxicity [J]. Photodiagnosis Photodyn Ther, 2009,6 (2): 122-127.

[18] Chan PS, Koon HK, Wu ZG, et al. Role of p38 MAPKs in hypericin photodynamic therapy-induced apoptosis of nasopharyngeal carcinoma cells [J]. Photochem Photobiol, 2009,85(5): 1207-1217.

[19] Sizhong Z, Xiukung G, and Yi Z. Cytogenetic studies on an epithelial cell line derived from poorly differentiated nasopharyngeal carcinoma [J]. Int J Cancer, 1983,31(5):587-590.

[20] Moore A, Donahue CJ, Bauer KD, et al. Simultaneous measurement of cell cycle and apoptotic cell death [J]. Methods Cell Biol, 1998,57:265-278.

[21] Guo C, Pan ZG, Li DJ, et al. The expression of p63 is associated with the differential stage in nasopharyngeal carcinoma and EBV infection [J]. J Transl Med, 2006,4: 23.

[22] Haworth P, Hess FD. The Generation of Singlet Oxygen $(\mathrm{O}(2))$ by the Nitrodiphenyl Ether Herbicide Oxyfluorfen Is Independent of Photosynthesis [J]. Plant Physiol, 1988,86(3):672-676.

[23] Kraljic I. Detection of singlet oxygen and its role in dye-sensitized photooxidation in aqueous and micellar solutions. Biochimie, 1986, 68:807-811.

[24] Gatenby RA, Taylor DD. Suppression of wound healing in tumor bearing animals as a model for tumor-host interaction: mechanism of suppression [J]. Cancer Res, 1990,50(24):7997-8001.

[25] Colombini, M. Purification of VDAC (voltage-dependent anionselective channel) from rat liver mitochondria [J]. J Membr Biol, 1983,74(2):115-121.

[26] Pastorino JG, Shulga N, and Hoek JB. Mitochondrial binding of hexokinase II inhibits Bax-induced cytochrome $\mathrm{c}$ release and apoptosis [J]. J Biol Chem, 2002,277(9):7610-7618.

[27] Kumarswamy R and Chandna S. Putative partners in Bax mediated cytochrome-c release: ANT, CypD, VDAC or none of them? [J]. Mitochondrion, 2009,9(1):1-8.

[28] Yousefi S, Simon HU. Autophagy in cancer and chemotherapy. Results Probl Cell Differ, 2009,49:183-190.

[29] Kabeya Y, Mizushima N, Yamamoto A, et al. LC3, GABARAP and GATE16 localize to autophagosomal membrane depending on form-II formation [J]. J Cell Sci, 2004,117(Pt 13):2805-2812.

[30 ] Von Ahsen O, Waterhouse NJ, Kuwana T, et al. The "harmless" release of cytochrome c [J]. Cell Death Differ 2000,7:1192-1199.

[31] Colombini M. Measurement of VDAC permeability in intact mitochondria and in reconstituted systems [J]. Methods Cell Biol, 2007,80:241-260.

[32] Brenner C and Kroemer G. Apoptosis. Mitochondria--the death signal integrators [J]. Science, 2000,289(5482):1150-1151.

[33] Sun J, Li ZM, Hu ZY, et al. Apogossypolone inhibits cell growth by inducing cell cycle arrest in U937 cells [J]. Oncol Rep, 2009,22(1): 193-198.

[34] Sattler M, Verma S, Shrikhande G, et al. The BCR/ABL tyrosine kinase induces production of reactive oxygen species in hematopoietic cells [J]. J Biol Chem, 2000,275(32):24273-24278.

[35] Cerimele F, Battle T, Lynch R, et al. Reactive oxygen signaling and MAPK activation distinguish Epstein-Barr Virus (EBV)-positive versus EBV-negative Burkitt's lymphoma [J]. Proc Natl Acad Sci U S A, 2005,102(1):175-179.

[36] Sastre-Serra JJ, Valle AA, Company MM, et al. Estrogen downregulates uncoupling proteins and increases oxidative stress in breast cancer [J]. Free Radic Biol Med, 2010,48(4):506-512.

[37] Pelicano H, Feng L, Zhou $Y$, et al. Inhibition of mitochondrial respiration: a novel strategy to enhance drug-induced apoptosis in human leukemia cells by a reactive oxygen species-mediated mechanism [J]. J Biol Chem, 2003,278(39):37832-37839.

[38] Khaitan D, Dwarakanath BS. Endogenous and induced oxidative stress in multi-cellular tumour spheroids: implications for improving tumour therapy [J]. Indian J Biochem Biophys, 2009,46 (1): 16-24.

[39] Conklin KA. Chemotherapy-associated oxidative stress: impact on chemotherapeutic effectiveness [J]. Integr Cancer Ther, 2004,3(4): 294-300.

[40] Rigby SE, Heathcote P, Evans MC, et al. ENDOR and special TRIPLE resonance spectroscopy of QA.- of photosystem 2 [J]. Biochemistry, 1995,34(37):12075-12081.

[41] Wagner JR, van Lier JE, Johnston LJ. Quinone sensitized electron transfer photooxidation of nucleic acids: chemistry of thymine and thymidine radical cations in aqueous solution [J]. Photochem Photobiol, 1990,52:333-343.

[42] Alegria AE, Ferrer A, Sepulveda E. Photochemistry of watersoluble quinones. Production of a water-derived spin adduct [J]. Photochem Photobiol, 1997,66:436-442.

[43] Wu RW, Chu ES, Yow CM, et al. Photodynamic effects on nasopharyngeal carcinoma (NPC) cells with 5-aminolevulinic acid or its hexyl ester [J]. Cancer Lett, 2006,242(1):112-119.

[44] Du HY, Olivo M, Tan BK, et al. Hypericin-mediated photodynamic therapy induces lipid peroxidation and necrosis in nasopharyngeal cancer [J]. Int J Oncol, 2003,23(5):1401-1405.

[45] Naim R. Photodynamic therapy using m-THPC (Foscan). Treatment of head and neck squamous cell carcinoma [J]. HNO, 2008,56(5):490-492.

[46] Puizina-Ivic N, Zorc H, Vanjaka-Rogosic L, et al. Fractionated illumination improves the outcome in the treatment of precancerous lesions with photodynamic therapy [J]. Coll Antropol, 2008,32 (Suppl 2):67-73.

[47] Wu SX, Chua DT, Deng ML, et al. Outcome of fractionated stereotactic radiotherapy for 90 patients with locally persistent and recurrent nasopharyngeal carcinoma [J]. Int J Radiat Oncol Biol Phys, 2007,69(3):761-769. 